\section{Management of traumatic wounds in the Emergency Department: a secondary publication}

\author{
Carolina Prevaldi, ${ }^{1}$ Ciro Paolillo, ${ }^{2}$ \\ Carlo Locatelli, ${ }^{3}$ Giorgio Ricci, ${ }^{4}$ \\ Fausto Catena, ${ }^{5}$ Luca Ansaloni, ${ }^{6}$ \\ Gianfranco Cervellin ${ }^{7}$
}

'Emergency Department, San Donà di Piave Hospital, San Donà di Piave (VE);

2Emergency Department, University

Hospital of Udine, Udine; ${ }^{3}$ Institute of

Toxicology, Maugeri Foundation, Pavia;

${ }^{4}$ Emergency Deparment, University

Hospital of Verona, Verona; ${ }^{5}$ Emergency

Surgery Department, University Hospital

of Parma, Parma; ${ }^{6}$ Emergency Surgery

Department, Hospital of Bergamo,

Bergamo; ' Emergency Department, University Hospital of Parma, Parma, Italy

\section{Abstract}

Traumatic wounds are among the most common problems leading people to the Emergency Department (ED), accounting for approximately $5.4 \%$ of all the visits, and up to $24 \%$ of all the medical lawsuits. In order to provide a standardized method for wound management in the ED, we have organized a workshop, involving several Italian and European experts. Later, all the discussed statements have been submitted for external validation to a multidisciplinary expert team, based on the so-called Delphi method. Eight main statements have been established, each of them comprising different issues, covering the fields of wound classification, infectious risk stratification, tetanus and rabies prophylaxis, wound cleansing, pain management, and suture. Here we present the results of this work, shared by the Academy of Emergency Medicine and Care and the World Society of Emergency Surgery.

\section{Introduction}

Traumatic wounds are among the most common problems leading people to the Emergency Department (ED), and account for approximately $5.4 \%$ of all the visits. ${ }^{1,2}$ The ED represents the most available facility for wound care, due to the 24-hour free access and the decreasing primary care availability. As such, provision for effective and safe wound care will continue to be a priority for Emergency Physicians (EPs). Moreover, traumatic wounds have been historically a major source of litigation against EPs, accounting for up to $24 \%$ of all the medical lawsuits, mainly due to missed identification and treatment of tendon or nerve injuries, or to infection and/or presence of foreign bodies. ${ }^{2}$ Hence, although most wounds will heal without any treatment, a prompt and careful repair of these injuries reduces infection and scarring, thus improving the patient's satisfaction and avoiding significant additional costs. ${ }^{1}$ However, in current clinical practice several different approaches to traumatic wounds are still practiced, due to cultural gaps, myths and local traditions.

One of the specific goals of the third European Union (EU) program in the health care area, years 2014-2020, is to improve access to a skilled, standardized and safe health care for EU citizens, thus improving the quality of health care and patient safety. According to these objectives we have organized a workshop aimed to share knowledge and experiences in the field of wound care, involving several Italian and European experts. The workshop was settled in Venice, in October 2014. Later, all the discussed statements have been submitted for external validation to a multidisciplinary expert team, as described in the methods. On the basis of the results of this complex and time-consuming work, the Academy of Emergency Medicine and Care (AcEMC) and the World Society of Emergency Surgery (WSES) have decided to build, write and spread a multidisciplinary position statement on the management of traumatic wounds in the ED.

The main purposes of the present work are: i) to assess the current scientific evidence on the subject; ii) to draw up a multidisciplinary consensus document aimed to establish a standardized and correct method of management of traumatic wounds in the ED; iii) help clinicians in the clinical risk stratification, to improve diagnostic and therapeutic appropriateness as well as the cost-benefit ratio, to reduce clinical errors, and to increase patient satisfaction; iv) provide an opportunity for research and educational initiative.

\section{Opinion Report}

We have decided to use a modified Delphi method, that is a structured communication technique, originally developed as a systematic, interactive forecasting method which relies on a panel of experts., ${ }^{3,4}$ The experts answer to one ore more questionnaires in two or more rounds. After each round, a facilitator provides an anonymous summary of the experts' forecasts from the previous round as well as the reasons they provide for their judgments. Thus, experts are encouraged to revise their
Correspondence: Carolina Prevaldi, Emergency Department, San Donà di Piave Hospital, Piazza De Gasperi 5, 30027 San Donà di Piave (VE), Italy. Tel: +39.0421 .227111 .

E-mail: cprevaldi@fastwebnet.it

Key words: Traumatic wounds; Infection; Foreign body; Tetanus; Rabies.

Note: writing committee members and external referee are reported in the Appendix.

Secondary publication: this article has been also published in World J Emerg Surg 2016;11:30. DOI: 10.1186/s13017-016-0084-3

Funding: the work has been made possible with the contribution of the Progetto Mattone Internazionale of the Italian Ministry of Health.

Received for publication: 1 April 2016.

Accepted for publication: 4 April 2016.

This work is licensed under a Creative Commons Attribution 4.0 License (by-nc 4.0).

CCopyright C. Prevaldi et al., 2016

Licensee PAGEPress, Italy

Emergency Care Journal 2016; 12:5906

doi:10.4081/ecj.2016.5906

earlier answers in light of the replies of other members of the panel. It is expected that during this process the range of differences of the answers will decrease and the group will converge towards the correct answer. Finally, the process is stopped after a pre-defined stop criterion (e.g., number of rounds, achievement of consensus, stability of results) and the mean or median scores of the final rounds determine the results. ${ }^{5}$

The Delphi method is based on the principle that forecasts or decisions obtained from a structured group of individuals are more accurate than those from unstructured groups. ${ }^{6}$ The name Delphi derives from the Oracle of Delphi, thus carrying in itself a somewhat mythical nuance. However, the method was developed at the beginning of the Cold War to forecast the impact of technology on warfare. ${ }^{6}$ One of the key characteristics of the method relies on the anonymity of the participants. As such, usually all participants remain anonymous, at least until the completion of the final report. This prevents the authority, personality, or reputation of some participants from dominating others in the process. Another important key characteristic is the regular feedback given to the participants, so that they can know comments on their own forecasts, as well as the responses of others, and the progress of the panel as a whole. The last key characteristic relies on the role of the facilitator, i.e. the person coordinating the group. He/she facili- 
tates the responses of their panel of experts, collects and analyzes them, thus identifying the conflicting viewpoints. If consensus is not reached, the process continues through thesis and antithesis, to gradually work towards synthesis, and building consensus.

To build this document we have composed a multidisciplinary panel consisting of EPs and surgeons, as well as other experts in different fields, coming from different countries. The study, which lasted about four months, was divided into two different phases. In both phases a dedicated questionnaire was sent by email to each member of the panel. In the first phase, there were three rounds. After that, consensus was reached in eight of the topics addressed. As such, in the second step it was considered as appropriate to repeat the round in order to try to reach consensus on all the addressed issues. The external validation of the document was reached organizing a two days' workshop, inviting a group of European experts to discuss and validate the statements. ${ }^{7,8}$

As such, the first step was based on a series of key questions, as reported in Table 1.

\section{Definitions}

At the end of the work the panel and the referees have reached an agreement on the following definitions of traumatic wounds. Traumatic wound is a wound or laceration of traumatic origin with no evidence of macroscopic contamination or signs of active infection (and likely low probability of infection). Dirty traumatic wound is a wound or laceration of traumatic origin macroscopically contaminated. Among these wounds we include those with simultaneous perforation of a viscus; with presence of devitalized tissues; with foreign bodies; those that occurred in a contaminated environment (dung, marshes); animal bites; puncture wounds; wounds with a delayed treatment. Infected traumatic wound is a wound or laceration of traumatic origin with signs of infection (secretions) ${ }^{9-13}$

After completing this step, the panel reached consensus on a series of statements concerning the management of traumatic wounds. For each statement, selected references are provided. The statements are as listed below.

\section{Statements}

\section{Statement 1}

All traumatic wounds are to be considered contaminated at presentation in ED.

\section{Statement 2}

It is useful to provide an initial stratification of the risk of infection for all the traumatic wounds. The risk assessment should be based on the following: i) type of wound; ii) location of the wound; iii) characteristics of the wounded patient. With the aim of simplifying and optimizing the management of patients in the ED, the following fields of stratification of the risk of infection were identified: type of wound, location of the wound, and characteristics of the patients. In Tables 2-4 the suggested items for risk assessment are summarized. Sub-statements of Statement 2 are: avoid antibiotic administration in low risk wounds (for all three variables considered) (2A); consider antibiotic administration when one or two high risk variables are present (2B); if the decision to avoid antibiotic administration in high risk wounds is made the reason must always be clearly stated (2C); in every wound consider the risk of tetanus according to the patient's immunization status (2D).

\section{Statement 3}

It is useful to provide antibiotic prophylaxis (i.e., a preventive administration of an antibiotic before the emergence of an infection with the aim to prevent it). It is desirable to implement prophylactic antibiotics in selected cases of wounds at high risk of infection. Sub-statements of Statement 3 are: avoid antibiotic prophylaxis in a non-macroscopically contaminated wound, well vascularized, at low risk of infection (according to statements 2) (3A); antibiotic prophylaxis should be considered in grossly contaminated wounds and in cases at high risk of infection (according to statement 2) depending on the epidemiological criteria of antibiotic resistance in the area. In high risk wounds (all three variables considered) the EP should explain clearly the reason for avoiding the antibiotic administration (3B). ${ }^{14-19}$

\section{Statement 4}

The assessment of tetanus immunization status in every traumatic wounded patient who arrives at the ED is desirable. Sub-statements of Statement 4 are: all traumatic wounds are potentially at risk for tetanus infection (4A); the assessment of tetanus immunization status of patients should be performed through a thorough history and consultation of documentation confirming vaccination/booster, and eventually using a diagnostic quick test in doubtful cases (4B); items to be considered as doubtful (i.e., cases for which it is not possible to determine the immunization status of the patient: patient who does not remember the date of the last booster; patient unconscious, intoxicated or cognitively impaired; patient who does not understand your language; patient who, presumably, has never carried out a complete vaccination course) (4C); access to vaccination data and the availability of a rapid diagnostic test for assessing the status of tetanus immunization permit to streamline costs and to act with greater appropriateness (4D)..$^{20-23}$

\section{Statement 5}

It is desirable that in any ED the first administration of rabies vaccine (for at least two patients) is available. Doses sufficient for full courses of rabies immunoglobulin treatment for two patients should be available in Poison Control Centers and in $2^{\text {nd }}$ level EDs (at least 1 for every 5 million inhabitants and at least 1 in each major island). ${ }^{24,25}$

\section{Statement 6}

A proper and timely implementation of procedures and methods for preventing infection in any traumatic wound is desirable. The identified methods of preventing infection are the

Table 1. Questions submitted to the experts.

1 Can you define clean a traumatic wound in the setting of the Emergency Department?

2 What is your approach to the prophylaxis of wounds with a high risk of infection (e.g., bites, wounds of the hand/foot...)?

3 Do signs predictive of risk of infection exist, and, if yes, how reliable are they?

4 Your opinion on methods of prevention of infection: irrigation, closure technique, antibiotic prophylaxis

5 In such wounds do you consider appropriate to assess the status of immunization against tetanus?

6 Do you consider appropriate the classification of traumatic wounds as clean wound not be tetanigenic?

7 Have you ever had difficulties during the anamnesis to assess the state of tetanus vaccine of injured patients who present to the Emergency Department?

8 Since only $15 \%$ of patients present with documented data on vaccinations and health registry is rarely accessible from the Emergency Room, in the absence of data, do you trust the patient's history on their vaccination status?

9 If you had to provide a quick diagnostic test to evaluate immediately and with certainty immunization status of injured patients on tetanus, would you consider it useful in the Emergency Department to improve the appropriateness of tetanus immunoprophylaxis and management of patients? 
Table 2. Infection risk assessment based on type of wound.

\begin{tabular}{ll}
\hline Straight stab wounds & Low risk \\
Tears/bruises/contusion wounds & High risk \\
\hline Puncture wounds & High risk \\
Wound with crush injuries & High risk \\
\hline Bite wounds & High risk \\
Wounds contaminated with feces & High risk \\
\hline Wounds contaminated with soil and dirt, or mineral oil & High risk \\
Wounds with the presence of foreign bodies & High risk \\
\hline Wounds with edge diastasis & High risk \\
Engagement of deep tissues, exposed fracture & High risk \\
\hline
\end{tabular}

Table 3. Infection risk assessment based on the location of the wound.

\begin{tabular}{ll}
\hline Well vascularized tissue (head, neck, scalp) & Low risk \\
High concentration of commensal flora (oral mucosa, genitals, armpits) & High risk \\
\hline Poorly vascularized (hand, foot, lower and upper limb) & High risk \\
\hline
\end{tabular}

Table 4. Infection risk assessment based on the characteristics of the patient.

\begin{tabular}{ll} 
Children & Low risk \\
Youngsters & Low risk \\
\hline Adults & Low risk \\
Elders ( $>65$ years) & High risk \\
\hline Immunocompromised (treated with steroids, immunosuppressive agents, & High risk \\
splenectomised, human immunodeficiency virus, ...) & \\
Vascular disease & High risk \\
\hline Diabetes & High risk \\
\hline
\end{tabular}

following. First, irrigation using appropriate security safeguards. Irrigation can be performed with saline (or tap water), with high pressure if necessary, according to the degree of contamination of the wound and the anatomic location (6A). Second, search for foreign bodies. Beside an accurate visual inspection, X-rays, computed tomography or ultrasound examination should be taken into consideration (6B). Third, suture technique (avoid shaving of hair; with simple stitches, always after irrigation; the intradermal suture should be avoided in most cases; if the risk of infection is high suture may be delayed) (6C)..$^{26-28}$

\section{Statement 7}

All the wounds of the hand should be carefully evaluated, considering them at high risk of error. Sub-statements of Statement 7 are: any traumatic injury of the hand should be considered for a possible tendon injury, especially if located on the volar or dorsal side (7A); any traumatic injury of the hand should be considered for a nerve injury, especially if located on the lateral side of the fingers (7B); a physical examination should be performed in any traumatic injury of the hand to check for any eventual tendon or nerve damage before performing the anaesthesia (7C); in every traumatic injury of the hand treated in the ED the possibility of performing a follow-up should be considered (7D). ${ }^{29-38}$

\section{Statement 8}

It is a priority to treat pain in traumatic wounds in all patients who attend to the ED. Several different protocols for the pain management are available, both pharmacological and non-pharmacological. Oral, local, intravenous, intra-nasal, and respiratory way (i.e., nitrous oxide) may be taken into consideration. ${ }^{39}$

\section{Conclusions}

We consider our work as a starting point and networking opportunity for participation in the forthcoming call funding programs in health care. In addition, the shared document (position paper) validated during the workshop with the precious contribution of international experts, intends to contribute to policy and health priorities in the European and interna- tional areas.

This article has been also published in World J Emerg Surg 2016;11:30 (DOI: 10.1186/s13017-016-0084-3). ${ }^{40}$

\section{References}

1. Hollander JE, Singer AJ. State of the art laceration management. Ann Emerg Med 1999;34:356-67.

2. Singer AJ, Hollander JE, Quinn JV. Evaluation and management of traumatic lacerations. New Engl J Med 1997;337: 1142-8.

3. Kung J, Miller RR, Mackowiak PA. Failure of clinical practice guidelines to meet Institute of medicine standards. Two more decades of little, if any, progress. Arch Intern Med 2012;172:1628-33.

4. Shaneyfekt T. In guidelines we cannot trust. Comment on "failure of clinical practice guidelines to meet institute of medicine standards". Arch Intern Med 2012;172:1633-4.

5. Rowe G, Wright G. The Delphi technique as a forecasting tool: issues and analysis. Int J Forecasting 1999;15:353-75.

6. Rowe G, Wright G. Expert opinions in forecasting. Role of the Delphi technique. In: Armstrong JS, ed. Principles of forecasting: a handbook of researchers and practitioners. Boston, MA: Kluwer Academic Publishers; 2001.

7. Dalkey N, Helmer 0. An experimental application of the Delphi method to the use of experts. Manage Sci 1963;9:458-67.

8. Linstone HA, Turoff M. The Delphi method: techniques and applications. Reading, MA: Addison-Wesley; 1975.

9. Edlich RF, Rodeheaver GT, Thacker JG, et al. Revolutionary advances in the management of traumatic wounds in the emergency department during the last 40 years: part I. J Emerg Med 2010;38:40-50.

10. Edlich RF, Rodeheaver GT, Thacker JG, et al. Revolutionary advances in the management of traumatic wounds in the emergency department during the last 40 years: part II. J Emerg Med 2010;38:201-7.

11. Quinn JV, Polevoi SK, Kohn MA. Traumatic lacerations: what are the risks for infection and has the 'golden period' of laceration care disappeared? Emerg Med J 2014;31:96-100.

12. Heggers JP. Assessing and controlling wound infection. Clin Plast Surg 2003;30: 25-35.

13. Lazarus GS, Zenilman GM. Wound microbiology: tabula rosa, a blank slate. Wound Repair Regen 2011;19:531.

14. Shaw TJ, Martin P. Wound repair at a glance. J Cell Sci 2014;122:3209-13. 
15. Hollander JE, Singer AJ, Valentine SM, et al. Risk factors for infection in patients with traumatic lacerations. Acad Emerg Med 2001;8:716-20.

16. Garcia-Gubern CF, Colon-Rolon L, Bond MC. Essential concepts of wounds management. Emerg Med Clin N Am 2010;28: 951-67.

17. Caldwell MD. Wound surgery. Surg Clin N Am 2010;90:1125-32.

18. Cooke J. When antibiotics can be avoided in skin inflammation and bacterial colonization: a review of topical treatments. Curr Opin Infect Dis 2014;27: 125-9.

19. Zehtabchi $S$. The impact of wound age on the infection rate of simple lacerations repaired in the emergency department. Injury 2012;43:1793-8.

20. Abbate R, Angelillo IF. Appropriate tetanus prophylaxis practices in patients attending Emergency Departments in Italy. Vaccines 2008;26:3634-9.

21. Talan DA, Abrahamian FM, Moran GJ, et al. Tetanus immunity and physician compliance with tetanus prophylaxis practices among emergency department patients presenting with wounds. Ann Emerg Med 2004;43:305-14.

22. Elkharrat D, Espinoza P, De la Coussaye J, et al. Inclusion of a rapid test in the current Health Ministry Guidelines with the purpose of improving anti-tetanus prophylaxis prescribed to wounded patients presenting at French Emergency Departments. Med Mal Infect 2005;35: 323 8.
23. Stubbe M, Mortelmans LJ, Desruelles D, et al. Improving tetanus prophylaxis in the emergency department: a prospective, double-blind cost-effectiveness study. Emerg Med J 2007;24:648-53.

24. Singer AJ, Dagum AB. Current management of acute cutaneous wounds. New Engl J Med 2008;359:1037-46.

25. Weiss EA, Oldham G, Lin M, et al. Water is a safe and effective alternative to sterile normal saline for wound irrigation prior to suturing: a prospective, double-blind, randomised, controlled clinical trial. Brit Med J 2013;3:e5001504.

26. Perelman VS, Francis GJ, Rutledge T, et al. Sterile versus nonsterile gloves for repair of uncomplicated lacerations in the emergency department: a randomized controlled trial. Ann Emerg Med 2004;43:36270.

27. ACEP. Clinical policy for the initial approach to patients presenting with penetrating extremity trauma. Ann Emerg Med 1999;33:612-36.

28. Amirtharajah M, Lattanza L. Open extensor tendon injuries. J Hand Surg-Am 2015; 40:391-7.

29. Chauhan A, Palmer BA, Merrell GA. Flexor tendon repairs: techniques, eponyms, and evidence. J Hand Surg-Am 2014; 39:184653.

30. Raval P, Khan W, Haddad B, et al. Bite injuries to the hand: review of the literature. Open Orthop J 2014;8:204-8.

31. Mehling IM, Arsalan-Werner A, Sauerbier M. Evidence-based flexor tendon repair.
Clin Plast Surg 2014;41:513-23.

32. Rrecaj S, Martinaj M, Murtezani A, et al. Physical therapy and splinting after flexor tendon repair in zone II. Med Arch 2014;68:128-31.

33. Neumeister MW, Amalfi A, Neumeister E. Evidence-based medicine: flexor tendon repair. Plast Reconstr Surg 2014;133: 122233.

34. Melamed E, Polatsch D. Partial lacerations of peripheral nerves. J Hand Surg-Am 2014;39:1201-3.

35. Ramirez EG, Hoyt KS. Assessment of acute hand injuries: part I. Adv Emerg Nurs J 2014;36:9-21.

36. Smith GA. Knife-related injuries treated in United States emergency departments, 1990-2008. J Emerg Med 2013;45:315-23.

37. Stepièn R, Szcz sny G. Current guidelines for management of severe hand injuries. Pol Orthop Traumatol 2014;79:82-7.

38. Tainter CR. An evidence-based approach to traumatic pain management in the emergency department. Emerg Med Pract 2012; 14:1-26.

39. Fosnocht DE, Swanson ER, Bossart P. Patient expectations for pain medication delivery. Am J Emerg Med 2001; 19:399-402.

40. Prevaldi C, Paolillo C, Locatelli C, et al. Management of traumatic wounds in the Emergency Department: position paper from the Academy of Emergency Medicine and Care (AcEMC) and the World Society of Emergency Surgery (WSES). World J Emerg Surg 2016;11:30. 